\title{
Gobernados por las deudas. El caso de los jóvenes adultos deudores de los créditos Corfo de Santiago de Chile
}

\author{
Lorena Pérez-Roa ${ }^{1}$
}

\author{
Recibido: 30/11/2016 / Revisado: 02/02/2017 / Aceptado: 14/11/2017
}

Resumen. En este artículo se propone analizar el caso del sistema de créditos estudiantiles de la Corfo desde una perspectiva de las teorías de la "gubernamentalidad". Utilizando los datos empíricos de una encuesta y de una serie de entrevistas realizadas por la autora, nuestro argumento plantea que esta modalidad de crédito favorece un proceso de "subjetivización" de los jóvenes deudores a través de tres elementos: en primer lugar, por los enclaves bancarios que este modelo de sistema de crédito crea para resguardar los riesgos financieros de transformar a "sujetos pobres" en "sujetos financieros". En segundo lugar, por las consecuencias de sumisión a un endeudamiento tentacular, al exigir anualmente avales o codeudores solidarios y, en tercer lugar, por los altos costos económicos y personales que para los deudores implica responder a sus compromisos financieros mes tras mes.

Palabras clave: gubernamentabilidad; créditos; educación universitaria; deudas; sujetos financieros.

\section{[en Governed by debt: the case of young adult debtors with Corfo loans in Santiago, Chile}

\begin{abstract}
The aim of this article is to analyse the case of the Corfo student loan system from the perspective of theories of governmentality. Using the empirical data from a survey and a series of interviews conducted by the author, our argument proposes that this form of credit encourages a process of subjectivisation of young debtors via three elements. The first element are the banking enclaves that this credit system model creates to safeguard the financial risks of transforming "poor subjects" into "financial subjects". Second are the consequences of submitting to pervasive indebtedness, with the annual requirement of guarantees or jointly liable co-debtors. Third are the high financial and personal costs that debtors face in having to meet their financial commitments from month to month.
\end{abstract}

Key words: governmentality; credits; university education; debt; financial subjects.

Sumario: Introducción. 1. El endeudamiento como sistema de financiamiento de la educación superior y el sistema de financiamiento universitario Corfo de pregrado. 2. Endeudar para educar: sobre los enclaves bancarios para acceder a la Universidad y la construcción social del "sujeto financiero". 3. Prácticas tentaculares de sumisión: el rol de los avales y codeudores solidarios. 4. La "rentabilidad" de ser un profesional-deudor Corfo y el disciplinamiento del "profesional endeudado". 5. Conclusiones. 6. Referencias bibliográficas.

Cómo citar: Pérez-Roa, L. (2018) Gobernados por las deudas. El caso de los jóvenes adultos deudores de los créditos Corfo de Santiago de Chile, en Cuad. trab. soc. 31(2), 455-466.

\section{Introducción}

Ser él o la primera profesional de la familia en Chile no solo representa un orgullo para los padres, sino que también para muchos es el reflejo de una sociedad que se dice más de- mocrática, en tanto el aumento sostenido de las expectativas de acceso de los jóvenes a la educación universitaria es un símbolo de apertura del sistema de educación superior chileno a segmentos de la población que antiguamente se encontraban excluidos de este

\footnotetext{
Trabajo Social, Universidad de Chile loperez@uchile.cl
} 
nivel educativo (Castillo y Cabezas, 2010). Las cifras en ese sentido son elocuentes. Si en 1990 la matrícula en establecimientos de educación superior era de poco más de doscientas mil personas, para el año 2016 esta cifra había ascendido a más de un millón cien mil personas (SIES, 2016). El acceso a la educación superior se valora, a su vez, por la capacidad de mejorar las oportunidades de los individuos en el mercado laboral y, por ende, de mejorar las remuneraciones futuras. En efecto, frases como "la educación es la gran palanca para la movilidad social" son frecuentes, tanto en los discursos públicos cómo en los mensajes movilizados en el espacio público por las universidades chilenas. Según la evidencia recogida por Simbürger (2013), la gran mayoría de universidades chilenas, utilizan agresivas estrategias de publicidad para vehiculizar el mensaje de que la educación superior mejora los ingresos y la empleabilidad futura de los individuos, presentándose más como universidades de emprendimiento individual que como espacios de enseñanza.

En efecto, diversos estudios han mostrado que las expectativas que los chilenos depositan en la educación social son muy altas (Araujo y Martuccelli, 2014; Pérez-Roa, 2014; Guzmán, Barozet, Méndez, 2017). Para muchos, "la educación permite salir de la pobreza, ascender social y ocupacionalmente, acceder a la cultura para ser tratado igual, acortar las distancias sociales y establecer vínculos con personas de otros grupos" (Guzmán, Barozet, Méndez, 2017, p. 100).

Ahora bien, ¿es que las expectativas puestas en la educación se cumplen independientemente de los medios que los jóvenes utilicen para financiar su proyecto educativo? Esta pregunta cobra particular relevancia en el caso chileno dado los elevados costos económicos de la educación superior que fueron sobrellevados antes de la implementación de la gratuidad el año $2015^{2}$ por los jóvenes y sus familias (Meller, 2011). De esta manera, para la mayoría de los jóvenes de clases medias y bajas el endeudamiento fue la principal estrategia de financiamiento de la educación superior (Meller, 2011; Urzúa, 2011). Los créditos Corfo, modalidad de crédito que exploramos en este trabajo, fueron, en efecto, los primeros créditos bancarios creados por el Estado para financiar proyectos educativos.

Entendiendo la importancia que tiene el explorar las políticas de financiamiento del acceso a la educación superior por la vía del endeudamiento, en este artículo proponemos una reflexión inspirada en los aportes de los estudios sobre la "gubernamentalidad" sobre el caso de los deudores de la Corfo de pregrado. En términos generales, los estudios de la "gubernamentalidad" o governamentality studies proponen un análisis del neoliberalismo como una forma específica de pensamiento contemporáneo que ha influenciado profundamente en la forma de pensar en la acción del Estado y sus modos de gobernanza. Este enfoque sostiene que el neoliberalismo más que favorecer la retirada del Estado a favor del mercado, favorece un modo de intervencionismo en los individuos aún más eficiente: son productores de ciudadanos-clientes quienes se autoperciben como individuos habilitados para mejorar sus vidas a través de sus decisiones individuales responsables e informadas en tanto consumidores de servicios y otros bienes (Schild, 2010). En este sentido, las "técnicas de gubernamentalidad" se caracterizan principalmente por transferir la responsabilidad del Estado relativa a los riesgos sociales al individuo (Lemke, 2001) y por producir una "subjetividad" que responsabiliza al individuo de sus actos y sus destinos (Luxton, 2010).

En ese sentido, el presente artículo sostiene que el sistema de financiamiento de la Corfo para el acceso a la universidad puede comprenderse como una "técnica de gubernamentalidad", en tanto que es una política pública de financiación del acceso a la universidad que no solo es un resultado de los cambios en la estructura económica chilena, sino también un intento gubernamental deliberado de crear consumidores atractivos para los nuevos mercados creados (González, 2017).

Nuestro argumento plantea que este proceso de subjetivización es observable específicamente en tres elementos de este sistema de financiamiento universitario: en primer lugar, en los enclaves bancarios que este modelo de sistema de crédito creó para resguardar los riesgos financieros de transformar a "sujetos pobres" en "sujetos financieros". En segundo lugar, por las consecuencias de sumisión a un

Desde al año 2015 se creó en Chile la política de gratuidad de acceso a la educación superior para el 50 por ciento de las familias de menores ingresos de la población cuyos miembros estudien en instituciones adscritas al beneficio. Referencia: www.gratuidad.cl 
endeudamiento tentacular que este instrumento crediticio provocó al exigir anualmente avales o codeudores solidarios $\mathrm{y}$, en tercer lugar, por los altos costos económicos y personales que implica para los deudores tener que responder a sus compromisos financieros mes tras mes.

Para desarrollar con más detalle este argumento, el artículo se divide en dos secciones. En la primera parte, presentaremos a grandes rasgos las principales características del sistema de financiamiento universitario en Chile en general y del sistema de créditos de la Corfo en particular. En este apartado se presentará además el encuadre metodológico que le dio origen a los datos empíricos que utilizaremos para este análisis. En la segunda parte, profundizaremos en aquellos elementos que nos permiten analizar los créditos Corfo como una técnica de "gubernamentalidad"; a saber: los enclaves financieros del crédito y la construcción de un sujeto financiero; las prácticas tentaculares de sumisión y el rol de los avales y el disciplinamiento del profesional-deudor. El articulo concluye con una síntesis del argumento expuesto y con algunas reflexiones sobre la necesidad de desarrollar una agenda de investigación sobre los procesos de endeudamiento y las relaciones financieras en Trabajo Social.

1. El endeudamiento como sistema de financiamiento de la educación superior y el sistema de financiamiento universitario Corfo de pregrado

El sistema de financiamiento de la educación superior en Chile tiene su origen en las grandes reformas impulsadas durante el gobierno militar, en el año 1981. Dicha reforma autorizó la creación de universidades privadas y de instituciones no universitarias - centros de formación técnica e institutos profesionales- $y$ rediseñó la estructura de financiamiento de las universidades, a través de la creación de dos instrumentos: un aporte fiscal directo (AFD), que funcionaba como un aporte base para las universidades públicas y privadas tradicionales, y un aporte fiscal indirecto o (AFI), que se introducía a modo de incentivo y que se repartía entre las instituciones de educación su- perior en proporción a la cantidad de alumnos de primer año matriculados y a sus respectivos puntaje en la prueba de selección de ingreso a la Universidad. Así, las universidades públicas y privadas existentes fueron impulsadas a recuperar parte de los costos de operación por medio del cobro de aranceles, creándose a su vez un fondo de ayudas estudiantiles - conocido hoy como Fondo Solidario de Crédito Universitario- para asistir a aquellos estudiantes que no pudieran pagar. Las nuevas universidades privadas, por el contrario, debían financiarse íntegramente por medio de los aranceles que cobrarían, sin que sus estudiantes contaran con acceso a créditos o ayudas estatales (Bernasconi y Rojas 2003, pp. 21-23). Para garantizar el acceso a la educación superior los gobiernos han desarrollado, a partir de 1981, un sistema de ayudas estudiantiles basado en créditos y becas. Este sistema, según las cifras del 2014, representó el 52 por ciento del total de los matriculados, del cual el 60 por ciento lo hacía mediante créditos (SIES, 2014).

Los créditos de pregrado de la Corfo pregrado - modalidad de crédito foco de nuestro interés- fueron el primer sistema de créditos para financiar proyectos en universidades no pertenecientes al Consejo de Rectores ${ }^{3}$. Creados en el año 1997 durante el gobierno de Eduardo Frei, se mantuvieron en vigencia hasta comienzos del 2012. Se trataba de un crédito administrado por los bancos comerciales a partir de fondos provistos y garantizados por el Estado, mediante la Cooperación de Fomento de la Producción (Corfo). En la práctica funcionaba como dos programas distintos, el crédito Corfo y el Subsidio Contingente Pregrado (Suco Pregrado). El primero consistía en prestamos con baja tasa de interés que la Corfo entregaba a las entidades financieras, con el propósito de que estas otorgaran créditos a los estudiantes, actuando estas últimas como intermediarios. Por su parte, el crédito conocido como Suco Pregrado consistía en una garantía que Corfo ofrecía a las entidades financieras, que se hacía efectiva una vez que los bancos agotaban las acciones judiciales de cobranza. La garantía cubría hasta el 50 por ciento del saldo impago del crédito con un límite de 500 $\mathrm{UF}^{4}$. Esto implicaba que la Corfo hacía presta-

\footnotetext{
El Consejo de Rectores de las Universidades chilenas es una persona jurídica de derecho público, de admintración autonóma, creado como un organismo de coordinación de la labor universitaria de la nación. Está integrado por los 25 Rectores de las 25 Universidades públicas y tradicionales del país. Fuente: http://www.consejoderectores.cl

4 Unidad de Fomento (UF) es una unidad financiera utilizada en Chile, reajustable de acuerdo con la inflación (medida según el Índice de Peso al Consumidor).
} 
mos a los bancos con una tasa del 3 por ciento, para que ellos prestaran luego a un máximo (extra) del 5 por ciento que, en caso de impago, estaba garantizado el 50 por ciento. El crédito era otorgado en UF con tasa de interés fija, de modalidad anual, renovable y financiaba hasta 200 UF anuales para el pago de la matrícula. El plazo máximo de pago era de 15 años por crédito solicitado. Según lo declarado por la Corfo, el objetivo de este crédito era "facilitar el financiamiento de estudios de pregrado en Chile otorgando una cobertura de garantía para favorecer el acceso al financiamiento de estudios de pregrado efectuados en universidades, institutos de formación técnica y centros de formación técnica en Chile" (Benavente et al., 2012, p. 86).

En efecto, la gran mayoría de las instituciones de educación superior eran elegibles y los criterios de selección de los postulantes, a diferencia de los otros tipos de crédito, no guardaban relación con su rendimiento académico. Lo importante, desde la lógica de los bancos prestamistas, era que el deudor les proveyera las garantías de pago necesarias, por ejemplo, contar con un aval o codeudor solidario. Al ser un sistema de crédito administrado por la banca privada, no existen cifras oficiales que permitan estimar ni la cantidad de estudiantes que financiaron sus proyectos académicos a través de los créditos de la Corfo ni el monto total adeudado. Solo en términos estimativos, según las cifras de la Superintendencia de Bancos e Instituciones Financieras de Chile (en adelante SBIF), al año 2010 existían 113.163 deudores de este sistema crediticio. Cabe señalar que los créditos de la Corfo de pregrado se eliminaron el año 2012.

Dada la escasa información oficial que existe sobre los deudores de la Corfo, para este análisis se utilizaran los datos empíricos elaborados en mi terreno de investigación doctoral, realizado durante el curso 2012-2013. En él se construyó y aplicó una encuesta que buscaba levantar información sobre el caso de los jóvenes adultos entre 25 y 35 años de la comuna de Santiago que fueron beneficiados, al menos, por un crédito Corfo para cursar sus estudios de pregrado. Esta cohorte de individuos fue elegida puesto que eran jóvenes que deberían haber accedido al sistema de educación superior antes de 2006, año en que se implementó el Crédito con Aval del Estado (CAE) ${ }^{5}$ y que, por edad al menos teóricamente, deberían haber comenzado su vida laboral profesional. La encuesta contó con 55 preguntas agrupadas en 5 ítems (antecedentes personales, trayectoria educativa, trayectoria laboral, trayectoria familiar, caracterización de la deuda). En términos de procedimiento, la encuesta fue enviada por correo electrónico a la totalidad de los casos de deudores de la ciudad de Santiago, registrados en la base de datos de la Asociación de Endeudados Corfo (560 casos), a través del servidor sondageonline.com. La opción de acceder a los jóvenes a través de la Asociación responde a la escasez de información oficial que existe sobre los acreedores de dicho crédito situación que impide la realización de otros tipos de muestreo. La encuesta se encontró accesible en línea desde el 15 de noviembre hasta el 15 de diciembre de 2012. En total respondieron 198 personas. Para efectos del análisis se excluyeron aquellos casos sin la información suficiente, lo que redujo la muestra a 156 casos. Se realizó un análisis descriptivo de la muestra estudiada, cuyos resultados fueron complementados con referencias a una serie de 16 entrevistas a jóvenes deudores de la Corfo, realizadas entre noviembre del 2012 y enero del 2013. Los detalles metodológicos y los principales resultados de estas entrevistas están detalladas en otro artículo (Pérez-Roa, 2014).

El crédito con aval del Estado (CAE) es un programa de prestamos de estudios ofrecidos en conjunto por el gobierno y los bancos comerciales. La institución de educación superior en la cual ingresa el alumno entrega una garantía parcial mientras el deudor estudia, para luego traspasarla al Estado desde la graduación hasta su total devolución. La retribución del crédito comienza 18 meses después de la graduación, y puede durar hasta un máximo de 20 años. Tienen acceso a este crédito los estudiantes de universidades acreditadas, y que hayan obtenido un puntaje mínimo de 475 en la Prueba de Selección Universitaria (PSU) 4 o un promedio de notas de la enseñanza media superior a 5,3 
Cuadro 1. Síntesis de la encuesta endeudados Corfo, Santiago, 2012

\begin{tabular}{ll}
\hline Universo & $\begin{array}{l}\text { Jóvenes deudores de un crédito Corfo } \\
\text { al menos, entre } 25 \text { y } 35 \text { años, habitantes } \\
\text { de la comuna de Santiago }\end{array}$ \\
Unidad de análisis & Individuos \\
Tamaño de la muestra & 560 \\
$\mathrm{~N}^{0}$ de respuestas recibidas & 198 \\
$\mathrm{~N}^{\mathrm{o}}$ de encuestas analizadas & 156 \\
Variables & Trayectoria educativa \\
& Trayectoria laboral \\
& Trayectoria familiar \\
& Trayectoria de endeudamiento \\
$\mathrm{N}^{0}$ total de preguntas & 55 \\
\hline
\end{tabular}

\section{Endeudar para educar: sobre los enclaves bancarios para acceder a la Universidad y la construcción social del "sujeto financiero"}

El sistema de créditos Corfo de pregrado se pensó como una forma de ayudar a los estudiantes universitarios de estratos de ingreso medio-alto, o a aquellos cuyos proyectos educativos fueran considerados de alta rentabilidad (medicina, ingenierías, etc.,). Sin embargo, una vez que este sistema comenzó a operar, los criterios de selección fueron prontamente definidos básicamente por los bancos que lo ofertaban (Donoso, 2009).

Si observamos los resultados de nuestra encuesta en relación con la proveniencia de las familias de origen de los jóvenes deudores constatamos que, a diferencia de lo estipulado en el diseño de la política, los beneficiarios reales del crédito Corfo se definen en su mayoría como provenientes de clase media (el 52,3 por ciento) o clase media-baja (el 35,9 por ciento) $\mathrm{Si}$ estos jóvenes y sus familias accedieron al crédito Corfo, fue porque la política pública la administraron las instituciones financieras, por ende los criterios de selección de los beneficiarios pasaron a tener un carácter principalmente comercial. Dicho de otra manera, al traspasar la administración a la banca privada, las preguntas sobre quiénes y qué tipo de proyecto educativo se redujeron a la pregunta sobre el riesgo financiero de los potenciales profesio- nales. De esta manera, y considerando que esta era una operación "arriesgada" desde un punto de vista financiero, las instituciones bancarias administradoras del crédito Corfo garantizaron su capital por medio de tres instancias. En primer lugar, mediante de la exigencia de un "aval" por lo menos, que correspondía a una persona natural o jurídica que voluntariamente asumía el compromiso de cumplir con la responsabilidad de pagar el crédito, en caso de que el estudiante no lo hiciera ${ }^{6}$. En segundo lugar, añadiendo el 5 por ciento adicional a la tasa de interés del 3 por ciento que Corfo cobró a las instituciones bancarias. En tercer lugar, mediante una garantía estatal que, tal y como explicamos anteriormente, se hacía efectiva una vez que los bancos agotaban las acciones judiciales de cobranza y cubría hasta el 50 por ciento del saldo impago del crédito con un límite de 500 UF. Todas estas garantías transformaron una operación financieramente arriesgada en un negocio rentable para las instituciones bancarias vinculadas al crédito Corfo $^{7}$. Desde una perspectiva de las teorías de la gubernamentalidad, esta maniobra financiera puede comprenderse como una transferencia de los riesgos de las responsabilidades del Estado, de proveer educación universitaria a los estudiantes. En efecto, el "riesgo financiero" fue traspasado directamente a los jóvenes, mediante el pago de altos intereses y de condicionar el crédito a una evaluación anual de las

\footnotetext{
Definición proporcionada por la Superintendencia de Bancos e Instituciones Financieras SBIF en su portal www.bancafacil.cl. En efecto, según los antecedentes correspondientes al período 2006-2008 del informe de financiamiento de la educación superior, elaborado por la SBIF, los índices de morosidad y de cobranza judicial asociado a los créditos Corfo se mantuvieron por debajo de los observados en las carteras de créditos de consumo. En junio de 2008, el índice de deudores morosos era del 14,9 por ciento, mientras que el porcentaje de deudores en proceso de cobranza judicial era del 3,2 por ciento.
} 
condiciones de solvencia de los jóvenes, sus familias y sus respectivos avales o codeudores solidarios.

Ahora bien, esta transferencia de riesgos no se concebía como un problema, en tanto el programa Corfo, al igual que toda la política de financiamiento de la educación superior en Chile, se había construido con base en el supuesto teórico de que obtener un título universitario permite obtener puestos de trabajo con mayores ingresos que permitirían pagar las deudas asociadas al crédito (Meller, 2007). Esta idea se fundamenta en la teoría del capital humano que supone que la educación es una inversión, en tanto el paso por ella garantiza un retorno económico. El problema es que este criterio hipotético-probabilístico no solo se asumió como verdad, sino que construyó toda una apología de lo que significaba esa verdad. En efecto, ser profesional para los participantes de nuestro estudio era una noción difusa, construida a partir de estas imágenes sociales que ubicaban a los profesionales como una categoría de persona que tiene mayores accesos al mercado de bienes y servicios:

Yo quería estudiar, yo quería estudiar para trabajar, ayudar en mi casa, tener mis cosas, porque en mi familia nadie tiene auto, no tenemos muchas cosas. Mi familia es de mucho esfuerzo, tenemos pocas cosas y vivimos con lo mínimo. Yo no tengo el plasma en la casa, yo no tengo el Wii, no tengo ninguna cuestión (....) Yo quería estudiar para ser algo po', cachai. No sé po, comprémonos, o vamos a la playa un mes (Laura, 28 años. Kinesióloga).

El sentido que Laura le da a ser profesional podría entenderse de la siguiente manera: un profesional es alguien que trabaja y que, gracias a la remuneración recibida, puede acceder a más cosas y mejores en el mercado de consumo. Al igual que para Laura, para la mayoría de los entrevistados la idea de ser un profesional era un proyecto asociado a mejores condiciones materiales de vida. Si ser profesional pudiere implicar tantos beneficios económicos, concretizando el anhelo de movilidad social individual, tomar un crédito para financiarlo dejaba de ser una maniobra arriesgada, y se vuelve un proyecto económico rentable.

Cuando reseñamos a que las condiciones del propio crédito promovieron un control de la subjetividad de los jóvenes deudores, nos referimos a las condiciones abusivas que el crédito impuso para crear "sujetos financieros" y los efectos perversos que tiene construir una "verdad social" a partir de construcciones teóricas abstractas, en tanto en ella no solo ignoran el carácter discrecional, arbitrario y discriminador de esas decisiones (Lemay, 2010), sino que también pretenden ignorar las condiciones de desigualdad en la cual se toma esta decisión "racional" de acceder al crédito (Gray, 2009).

\section{Prácticas tentaculares de sumisión: el rol de los avales y codeudores solidarios}

Tradicionalmente se entiende que la relación entre el acreedor y el deudor es una relación de confianza. En la medida en que el deudor -sujeto individualizado y perfectamente bien caracterizado - detenta una deuda con otro, se involucra en una relación social de confianza, marcada por la obligación de responder al compromiso financiero. Para Lazzarato (2011) el uso de la palabra "confianza" en el mercado crediticio esconde, bajo la apariencia de un reconocimiento del otro, un interés por acceder a las acciones pasadas, presentes y futuras de los deudores, en clave de solvencia. Es decir, se pretende evaluar si un individuo puede ser considerado como "sujeto crediticio", en la medida de que sus posesiones, sus comportamientos y sus valores permitan "confiar" en su capacidad futura de pago. En efecto, independientemente del tipo de crédito que se solicite, el procedimiento de evaluación es esencialmente el mismo: el agente de crédito recolecta la información del solicitante -identidad, estado civil, historia ocupacional, salarios de empleos, activos, pasivos, cuentas bancarias, entre otros- y verifica los datos con los que posee -en virtud de transacciones anteriores o de fuentes externas, como el historial crediticio del deudor- para finalmente traducir toda la información a un score que es atribuido al solicitante, con el fin de evaluar el riesgo que representa para el acreedor (Duhaime, 2003). Dicho score se construye principalmente a partir de datos "acreditables", es decir, medibles, cuantificables y "objetivables", como: contratos de trabajos, títulos de propiedades, títulos universitarios, etc. Requisitos que no necesariamente podían ser cumplidos por los jóvenes y sus familias, en tanto la gran mayoría de los jóvenes deudores del Corfo son la "primera generación de matriculados en la universidad". 
Sin embargo, tal y como ya lo hemos señalado, los bancos garantizaron su inversión a través de las exigencias de avales o codeudores solidarios. Desde el punto de vista de las instituciones financieras, el aval es una figura que les permite controlar la incertidumbre económica que implica el prestar dinero a alguien, sobre todo cuando ese alguien no posee bienes embargables en caso de no pago. Mientras que, desde el punto de vista de los jóvenes deudores Corfo, el aval no solo era la persona que les permitía que esa deuda se contrajera, sino también la principal razón por la cual la deuda se pagaba mes tras mes.

En efecto, conseguir un aval no era una tarea fácil, y exigía desplegar una serie de estrategias que, en el caso de muchos de los participantes de este estudio, implicaba salir a buscarlo fuera del núcleo familiar más cercano. Si uno observa la red de relaciones que sostiene cada uno de los créditos Corfo adquiridos por los jóvenes endeudados, aparecen familiares, amigos, colegas de trabajo, amigos de los padres, quienes conforman una red intersubjetiva de personas que desborda la tradicional triada acreedora-deudor-aval. Un joven deudor, por ejemplo, nos explicaba que el primer año le pidió a un colega de trabajo que fuera su aval, pero al segundo año este no quiso seguir siéndolo, por lo que tuvo que recurrir a su jefa directa, quien, "de buena voluntad", lo ayudó durante dos años. Luego, el último año le pidió ser aval a otra persona de su trabajo, porque no quiso volverle a pedir los papeles que acreditarán solvencia a su jefa y el banco le negó la renovación del crédito, cuando él cursaba su último año de carrera, razón por la cual se vio obligado a aumentar su ya extensa carga de trabajo para poder costear la matricula. Sin embargo, a la fecha de nuestro encuentro aún no había logrado juntar el dinero suficiente, para pagar sus créditos y los costos de la matrícula. Dicho de otra manera, este joven en tres años contrajo una deuda con el banco, con su jefa $\mathrm{y}$ un colega de trabajo y, a pesar del monto adeudado, aún no sabía si iba a poder costear su último año de carrera.
De esta manera, cuando decimos que las instituciones bancarias desarrollaron prácticas tentaculares de sumisión, nos referimos justamente a este tipo de prácticas de extensión de su poder de sometimiento a un crédito abusivo a una red extendidas de personas, sin ni siquiera asegurar con ello, el financiamiento de todo el proceso de estudios de los jóvenes.

\section{La "rentabilidad" de ser un profesional- deudor Corfo y el disciplinamiento del "profesional endeudado"}

La evidencia del comportamiento crediticio en las poblaciones universitarias señala que existiría una relación potencial entre las actitudes que los estudiantes tienen hacia el endeudamiento y las expectativas de su futuro laboral. Este supuesto implica asumir que el paso por la educación superior conlleva en sí mismo una rentabilidad económica que se materializa en un aumento considerable del valor del trabajo del profesional.

Ahora bien, si observamos la situación laboral de nuestros encuestados, del total de jóvenes, el 80,3 por ciento de ellos se encontraba trabajando en el momento de realización de la encuesta, mientras solo el 19,7 por ciento declaró no estar trabajando. En relación a la remuneración percibida, tal y como lo vemos en la Tabla 1, el 45,6 por ciento de los jóvenes que se encontraban trabajando percibían ingresos que iban desde los 450.000 pesos (1.000 dólares estadounidenses aproximadamente) a los 950.000 pesos (2.000 dólares estadounidenses aproximadamente). Si comparamos esa cifra con los datos nacionales que muestran que solo el 6,8 por ciento de la población en Chile obtiene más de 1.000 .000 pesos (2.000 dólares estadounidenses aproximadamente) mientras el 53,2 por ciento obtiene menos de 300.000 pesos (600 dólares estadounidenses aproximadamente) líquidos mensuales, podemos decir que nuestros encuestados se encuentra en una situación de privilegio en relación a los ingresos de la gran mayoría de los trabajadores chilenos. 
Tabla 1. Ingresos mensuales

\begin{tabular}{lll}
\hline \multicolumn{1}{c}{ Pesos chilenos } & \multicolumn{1}{c}{ \$ USD $(*)$} & \\
\hline Entre $\$ 0$ y $\$ 150.000$ & Entre 0\$ y $297 \$$ & $17,3 \%$ \\
Entre $\$ 150.001$ y $\$ 300.000$ & Entre $297 \$$ y $594 \$$ & $14,3 \%$ \\
Entre $\$ 300.001$ y $\$ 450.000$ & Entre $594 \$$ y $899 \$$ & $15,8 \%$ \\
Entre $\$ 450.001$ y $\$ 600.00$ & Entre $899 \$$ y $1188 \$$ & $21,8 \%$ \\
Entre $\$ 600.001$ y $\$ 950.000$ & Entre $1188 \$$ y $1881 \$$ & $23,8 \%$ \\
Entre $\$ 950.001$ y $\$ 1.500 .000$ & Entre $1881 \$$ y $2970 \$$ & $6,0 \%$ \\
Más de $\$ 1.500 .001$ & Más de $2970 \$$ & $1,5 \%$ \\
\hline
\end{tabular}

Fuente: Elaboración propia. Encuesta endeudados Corfo, Santiago. 2012 n= 133

Más allá de esta posición de privilegio, lo interesante a observar para este análisis es el peso que tiene el pago mensual del crédito en el salario declarado por los jóvenes participantes. Tal y como lo muestra la tabla 2 el 61,5 por ciento de los jóvenes deudores encuestados declaró que destinaba entre el 16 por ciento y el 30 por ciento del total de sus ingresos al pago del crédito Corfo.

Tabla 2. Porcentaje de su sueldo que destina al pago de la deuda

\begin{tabular}{ll}
\hline Menos del $15 \%$ & $5,5 \%$ \\
Entre un $16 \%$ y un $30 \%$ & $61,5 \%$ \\
Entre un $31 \%$ y un $45 \%$ & $11,9 \%$ \\
Entre un $46 \%$ y un $60 \%$ & $11,9 \%$ \\
Entre un $61 \%$ y un $75 \%$ & $6,4 \%$ \\
Entre un $76 \%$ y un $100 \%$ & $2,8 \%$ \\
Total (N 100 ) & $100 \%$ \\
\hline
\end{tabular}

Fuente: Elaboración propia. Encuesta endeudados Corfo, Santiago. 2012 n= 156

Si comparamos este porcentaje con la evidencia internacional que Meller (2011, pp.5173) proporciona, notamos que los jóvenes profesionales egresados de países como Alemania, Australia, Canadá, Reino Unido y Estados Unidos tienen obligaciones mensuales de pago de su crédito universitario entre el 2,6 por ciento y el 7 por ciento de su ingreso mensual. De esta manera, pagar entre el 16 por ciento y el 30 por ciento del salario solo en concepto de crédito universitario situaría a los jóvenes deudores Corfo con uno de los niveles de endeudamiento estudiantil presumiblemente más altos del mundo.
Ahora bien, a pesar de los altos costos asociados a esta modalidad de crédito, $\mathrm{y}$ al alto peso que implica en la vida de estos jóvenes y sus familias responder a este compromiso financiero, el 81,4 por ciento de los jóvenes encuestados en el momento de la aplicación de la encuesta tenía sus cuotas al día y solo el 18,6 por ciento declaró estar retrasado con el pago de su deuda. Las propias características de sujeción del comportamiento de los jóvenes deudores explican las razones por las cuales esta deuda se paga sagradamente mes tras mes. Ya sea por los avales involucrados o por los elevados costos personales y familiares que podría 
implicar no pagar la deuda la gran mayoría de los jóvenes paga sus deudas:

[El CORFO es para mí] una pesadilla, la razón por la cual la prometida movilidad social que se supone que obtendría al estudiar ha sido más negativa que nada. Una estafa por la cual nadie responde. A significado trabajar para solo pagar los créditos y no comprar nada que signifique una mejora en la calidad de vida propia y de los que me rodean. En resumen, un calvario. "Trabajar para pagar".

Esa es la sensación que tienen todos nuestros entrevistados. Ya sea porque las condiciones laborales a las que han accedido, siendo ya profesionales, no son lo "suficientemente" buenas como para poder responder a sus compromisos financieros, o por los elevados montos que pagan por concepto de crédito universitario, que en algunos casos superan los 600 dólares estadounidenses mensuales. Todos los entrevistados sienten que el crédito ha limitado sus posibilidades pasadas y presentes, como también las futuras.

El Corfo es el mejor anticonceptivo que existe, porque te tienes que planificar de tal manera que no te puedes salir de tus gastos (dice Consuelo, psicóloga, 29 años).

Efectivamente, las conductas futuras que los jóvenes proyectan se encuentran predispuestas en función del pago de la deuda. Ser o no ser madre-padre, independizarse o no, son proyectos evaluados y racionalizados a partir del estado de endeudamiento que se tiene, y de las proyecciones futuras -por lo tanto, inciertas-de reembolsarlo.

Siguiendo los argumentos de Foucault en general, y del nacimiento de la biopolitica en particular, Lazzarato (2011) sostiene que la deuda implica una producción de subjetividad especifica: aquella del hombre endeudado, cuya principal tarea consiste en ser garante de sus acciones, de su manera de vivir y hacerse responsable de ello. La deuda, a su juicio, secreta una moral propia, que introduce la idea de que todo quien no pueda asumir un "modo de vida" adecuado al rembolso de sus deudas (modos de consumo, empleo, gastos sociales, etc.,) es culpable de no honrar sus deudas. En este sentido, los altos costos que ha tenido pagar el crédito para los jóvenes y sus familias, $\mathrm{y}$ la obligación moral de pago que la estructura tentacular de este modelo de endeudamiento provocó generaron un disciplinamiento de las conductas presentes y futuras de los, ahora, profesionales endeudados.

\section{Conclusiones}

En este trabajo se ha propuesto analizar el caso del sistema de créditos estudiantiles de la Corfo desde una perspectiva de las teorías de la "gubernamentalidad". Nuestro propósito era explorar cómo esta modalidad de financiación de la política de acceso a la educación universitaria transformó en su actuar a los jóvenes provenientes de clase más pobres en "sujetos financieros" y con ello en "deudores educacionales" cuyas conductas deben adecuarse al pago de sus obligaciones crediticias. Utilizando los datos mi trabajo doctoral, hemos caracterizado tres elementos de este proceso de endeudamiento como una técnica de "gubernamentalidad":

- En primer lugar, analizamos cómo la política pública, a través de la creación de créditos como el Corfo, traslado los riesgos sociales a los individuos. De esta manera, se hizo responsable a los individuos y a su capacidad de transformarse en "sujeto financiero" de los riesgos financieros de la operación crediticia. En este sentido, exploramos como para los deudores esta operación "ariesgada" se justificaba en tanto el acceso a la universidad era significado como una promesa de movilidad social.

- En segundo lugar, describimos el peso que tuvo la exigencia del aval o codeudor solidario en los procesos de subjetivación de los jóvenes deudores. La extensión tentacular del poder de sumisión del crédito a una red de personas, sin ni siquiera asegurar con ello el financiamiento total del proyecto educativo del deudor principal, es a nuestro parecer una clara muestra del poder de subjetivización de esta modalidad de crédito. Los deudores, no solo comprometieron sus proyectos educativos y personales al contraer los créditos, sino que también se volvieron responsables de proteger al aval de los perjuicios que puede ocasionarle su morosidad. 
- En tercer lugar, mostramos cómo las condiciones poco favorables del crédito (tasa de interés, exigencia de aval, modalidad anual, garantía estatal, etc.) resultaron difíciles de sostener por los jóvenes y sus familias. Tal y como observamos en los resultados de nuestro análisis, los jóvenes deudores destinan un porcentaje superior al 16 por ciento de su salario exclusivamente al pago de sus compromisos crediticios. Cifra que sitúa a los jóvenes deudores de la Corfo con un nivel de endeudamiento estudiantil presumiblemente como uno de los más altos del mundo. Los costos de la deuda han obligado a los jóvenes deudores a adaptar su comportamiento a las exigencias de pago. En este proceso de "normalización de conductas" (Lazzarato, 2011) los jóvenes no solo se comprometen su cotidianeidad, sino también sus proyecciones futuras.

Finalmente, quisiéramos detenernos en la relevancia que tiene para las Ciencias Sociales, en general, y para el Trabajo Social, en particular, iniciar una agenda de investigación en esta línea. Los procesos de endeudamiento y de subjetivación de los deudores, se inscriben dentro de una política de conducción de conductas propias del neoliberalismo. Es una puerta de entrada para observar las implicancias que tiene en la vida cotidiana de los individuos inte- riorizar prácticas preformativas de autogobierno. No poder responder con los compromisos financieros, muchos lo viven como un fracaso individual. Asumir, desde el lugar del fracaso, su situación de endeudamiento, no solo tiene consecuencias en la manera en que estos jóvenes se representan a sí mismos, sino que también define las posibilidades de transformación que sean capaces de observar. Con una lectura feminista, una de las consecuencias de las políticas neoliberales de responsabilizar a los individuos es la generación de un estado de resignación o lack of vision (Braedley y Luxton, 2010), provocando que las personas se sientan responsables no solo de sus actos sino también de sus destinos, y generando, al mismo tiempo, un estado de pasividad en el cual los individuos sienten que no "pueden hacer nada" con la situación que los aqueja, transformándose en individuos inactivos políticamente (Pinto, 2010). De esta manera, iniciar una agenda de investigación en estos temas implica pensar no solo en construcciones analíticas que nos permitan salir de nociones binarias y moralizantes que inundan el campo del Trabajo Social, sino también pensar en nuevas formas de solidaridad y colaboración que nos permitan transitar desde una comprensión individualizada de la deuda, en la cual ellos son responsables absolutos de su situación, a una comprensión del endeudamiento estudiantil como un problema social cuyas respuestas dependen del conjunto de la sociedad.

\section{Referencias bibliográficas}

Araujo K. y Martuccelli, D. (2014). Beyond institutional individualism: Agentic individualism and the individuation process in Chilean society. Current Sociology, 62(1), EUA: Sage

Araujo, K y Martuccelli, D. (2012). Desafios Comunes: Retratos e la sociedad chilena y des sus individuos. Tomo 1 y 2. Santiago: LOM.

Barros, P. (2008). ¿Tres cuotas, precio contado? Instituto de Investigación en Ciencias Sociales, 81-89.

Bauman, Z. (2008). S'acheter une vie. París: J. Chambon.

Benavente, J., Álvarez, P., Tejeda, I., Hoces, F., Lima, J., Contreras, J., Trivelli, C., Valdivieso, I., Sánchez, G. y Medrano, P. (2012). Evaluación de impacto de las becas de educación superior - Informe final. Santiago de Chile: Verde e Intelis.

Bernasconi, A. y Rojas, F. (2004). Informe sobre la educación superior en Chile: 1980-2003. Santiago de Chile: Editorial Universitaria.

Bowen, William G., Matthew M. Chingos y McPherson, Michael S. (2009). Crossing the Finish Line: Completing College at America's Public Universities. Princeton: Princeton University Press.

Castillos, J. y Cabezas, G. (2010). Caracterización de jóvenes de primera generación en educación superior. Nuevas trayectorias hacia la equidad educativa. Calidad en la Educación, 32, 44-74. Disponible en: http://www.cned.cl/public/secciones/seccionpublicaciones/doc/67/cse_articulo900.pdf 
Denegri, M., Cabezas D., Del Valle C., González, Y. y Sepúlveda, J. (2012) Escala de Actitudes hacia el Endeudamiento: validez factorial y perfiles actitudinales en estudiantes universitarios chilenos. Universitas Psychologica 11(2), 497-509.

Donoso, S.D. (2009). Economía política del financiamiento de los estudios universitarios en Chile (19802010): debate de sus fundamentos. Innovar-Revista De Ciencias Administrativas y Sociales, 19(1). Diponible en: http://www.scielo.org.co/pdf/inno/v19s1/19s1a11.pdf

Duhaime, G. (2003). La vie à crédit: consommation et crise. Sainte-Foy, Québec, Canadá: Presses de l'Université Laval.

Ehrenberg, A. (1999). Le culte de la performance. París: Hachettes LittÈratures.

Guzmán, V., Barrozet, E. y Méndez, L. (2017). Legitimización y crítica a la desigualdad. Una aproximación pragmática. Revista Convergencia, 73, 87-112

Han, C. (2011). Symptoms of another life: Time, Possibility, and Domestic Relations in Chile's Credit Economy. Cultural Anthropology, 26(1), 7-32. doi: 10.1111/j.1548-1360.2010.01078.x

Instituto Nacional de la Juventud Gobierno de Chile. (2009) Endeudamiento juvenil. Resultados preliminares VI encuesta Nacional de Juventud. Recuperado de: http://slideplayer.es/slide/83988/

Lazarus, J. (2009). L'épreuve du crédit. sociétés contemporaines, 4(76), 17-39. doi: 10.3917/soco.076.0017

Lazzarato, M. (2011). La fabrique de l'homme endetté : essai sur la condition néolibérale. París: Amsterdam.

Lemay, V. (2000). Évaluation scolaire et justice sociale. Droit, éducation et société. Saint-Laurent: Renouveau pédagogique.

Lemke, T. (2001). 'The birth of bio-politics': Michel Foucault's lecture at the collëge de france on neo-liberal governmentality. Economy and society, 30(2), 190-207.

Lemke, T. (2004). «Marx sans guillemets : Foucault, la gouvernamentalité et la critique du néolibéralisme, Actuel Marx, 2(36), 13-26.

Malcom, L.E y Dowd A.C. (2012). The Impact of Undergraduate Debt on the Graduate School Enrollment of STEM Baccalaureates. The Review of Higher Education 35(2), 265-305.

Marambio, A. (2011). Bancarización, crédito y endeudamiento en los sectores medios. Disertación de Maestría en sociología. Universidad de Chile.

Marron, D. (2007). "Lending by numbers": credit scoring and the constitution of risk within American consumer credit. Economy and society, 36(1), 103-133.

Meller, P. (2011). Universitarios, ;el problema no es el lucro, es el mercado! Santiago: Uqbar Editores.

Ministerio de Educación, Gobierno de Chile. (2011). Estadísticas de la educación 2011. Recuperado de: http://centroestudios.mineduc.cl/index.php?t=96

Ministerio de Educación, Gobierno de Chile. (2012) Comisión de Financiamiento estudiantil. Recuperado de: http://200.6.99.248/ bru487cl/files/ComParedes.pdf

Núñez, J. y Miranda, L. (2009). La movilidad intergeneracional del ingreso y la educación en Chile. En: El arte de clasificar a los chilenos: Enfoques sobre los modelos de estratificación social en Chile. Santiago: Ed. UDP, serie políticas públicas.

OCDE y Banco Mundial. (2009). La educación superior en Chile. Recuperado de: http://www.oecd.org/ edu/Chile-EAG2014-Country-Note.pdf

Olavarría, M. y Allende, C. (2013). Student Debt and Access to Higher Education in Chile. Revista española de investigación sociológica, 141, 91-112.

Pérez Roa, L. (2104). El peso real de la deuda de estudios: La problemática de los jóvenes deudores del sistema de financiamiento universitario de la Corfo pregrado en Santiago de Chile. Archivos Analíticos de Políticas Educativas, 22(62).

Ruiz, C. y Boccardo, G. (2015). Los chilenos bajo el neoliberalismo. Clases y conflicto social. Fundación Nodo XXI y El desconcierto. Chile.

Salamanca, J. (2000). El crédito como instrumento para financiar el acceso y la mantención de los estudiantes en las instituciones de educación superior en Chile. Estudio de caso para obtener el grado de Magister en Gestión y Políticas Públicas, Universidad de Chile.

Schild, V. (2000). Neo-liberalism's New Gendered Market Citizens: The 'Civilizing' Dimension of Social Programmes in Chile. Citizenship Studies, 4(3), 275-305.

Simburguer, E. (2013). Moving through the city: visual discourses of upward social mobility in higher education adverstisement on public transport in Santiago de Chile. Visual Studies, 28(1), 67-77. Disponible en: http://dx.doi.org/10.1080/1472586X.2013.765219 
Superintendencia de Bancos e Instituciones Financieras. (2008). Financiamiento de Estudios de Educación Superior (Antecedentes 2006-2008). Santiago de Chile: Departamento de Estudios.

Urzúa, S. (2012). La rentabilidad de la educación superior en Chile: revisión de las bases de 30 años de políticas públicas. Estudios Públicos, 125, 1-52.

Zahler, R. (2008). Bancarización privada en Chile. Santiago de Chile: CEPAL. 